\title{
Prognostic value of the modified model for end-stage liver disease (MELD) score including albumin in acute heart failure
}

\author{
Shengen Liao ${ }^{\dagger}$, Xinyi Lu ${ }^{\dagger}$, lokfai Cheang, Xu Zhu, Ting Yin, Wenming Yao, Haifeng Zhang and Xinli Li* (i)
}

\begin{abstract}
Background: Liver and renal function evaluated by the model for end-stage liver disease (MELD) score, the MELD excluding the international normalized ratio (MELD_XI) score and the MELD including sodium (MELD_sodium) score have been considered predictors of adverse events for patients with acute heart failure (AHF). However, the prognostic value of the MELD including albumin (MELD_albumin) score in patients with AHF has not been assessed.

Methods: A total of 466 patients with AHF were prospectively evaluated. We compared the accuracy of the 4 MELD score formulas using the time-dependent receiver operating characteristic (ROC) curve and corresponding areas under the curve (AUC).

Results: During a median follow-up period of 34 months, 196 deaths occurred. In the fully adjusted Cox regression model, standardized hazard ratios with $95 \%$ confidence interval expressing the risk of all-cause mortality were 1.22 (1.06-1.40), 1.20 (1.04-1.39), 1.23 (1.06-1.42) and 1.21 (1.05-1.41) for MELD, MELD_XI, MELD_sodium and MELD_albumin scores, respectively. The MELD_albumin score showed the best prognostic accuracy $(A U C=0.658)$ for the prediction of long-term all-cause mortality, followed by the MELD_sodium score (AUC $=0.590$ ), the MELD score $(A \cup C=0.580)$, and the MELD_XI score $(A \cup C=0.544)$; the MELD_albumin score performs significantly more accurate than MELD and MELD_XI score for predicting the risk of all-cause mortality. Considering reclassification, MELD_albumin score increased the net reclassification improvement over and beyond MELD (13.1\%, $P=0.003)$, MELD_XI (14.8\%, $P=0.002)$, and MELD_sodium $(11.9 \%, P=0.006)$ scores for all-cause mortality.
\end{abstract}

Conclusions: The MELD_albumin score increases risk stratification of all-cause mortality over and beyond the MELD score and the other modified MELD scores in patients with acute heart failure.

Keywords: Model for end-stage liver disease, Mortality, Albumin, Acute heart failure

\section{Background}

Heart failure (HF) is a common disease and is associated with considerable morbidity and mortality worldwide [1]. With the increase in HF patients, HF has recently

\footnotetext{
*Correspondence: xinli3267@njmu.edu.cn

†'Shengen Liao and Xinyi Lu contributed equally to this work and should be considered co-first authors.

Department of Cardiology, Jiangsu Province Hospital and Nanjing Medical University First Affiliated Hospital, Guangzhou Road 300, Nanjing 210029, China
}

become a serious health problem [2]. Acute heart failure (AHF) is a complex syndrome characterized by worsening heart failure symptoms. The predominant clinical profile in most patients with AHF is congestion and hypoperfusion, which can lead to organ dysfunction and increase the risk of mortality.

Liver and renal dysfunction are often complicated in patients with acute heart failure [3]. Complicated interaction between heart, kidney, and liver has been an object of interest for a long time. In that sense, simple score 
capable of quantitating the severity of multiorgan failure is attractive. Biomarkers that reflect liver and kidney function are often used to predict adverse clinical outcomes in patients with AHF $[4,5]$. The model for endstage liver disease (MELD) score evaluating liver and renal function was considered a reliable predictor for the risk of adverse events in AHF patients [6]. Several studies also focused on the effects of modified MELD versions, such as the MELD excluding the international normalized ratio (MELD_XI) score [3, 7], and the MELD including sodium (MELD_sodium) score [8], on the prognosis of acute heart failure. However, the prognostic value of the MELD including albumin (MELD_albumin) score, which includes the serum value of albumin rather than the international normalized ratio, in AHF patients is still unknown. Moreover, it is largely unknown whether one of the MELD scores or its 3 modifications is superior for predicting the risk of mortality.

Accordingly, we aimed to assess the prognostic role of the MELD_albumin score in patients with AHF over a long-term follow-up. In addition, we further aimed to compare the accuracy of the MELD_albumin score with other well-established objective MELD and modified MELD scores for predicting the risk of death in patients with AHF.

\section{Methods}

\section{Study subjects}

We analyzed data from the registry study of AHF in China: the diagnostic standards, risk stratification, and clinical outcomes of acute heart failure (registration number: ChiCTR-ONC-12001944), which is a multicenter, observational, prospective cohort study. From March 2012 to June 2017, 590 patients with AHF were assessed for eligibility. Patients with end-stage renal dysfunction/hemodialysis and decompensated liver cirrhosis were excluded in our study. The diagnosis of AHF was based on clinical signs and symptoms and according to the guidelines, and each patient received standard clinical evaluation and guided recommended treatment [9]. Patients with severe vascular disease or coronary artery disease requiring urgent surgery $(n=46)$ or who withdrew informed consent $(n=2)$ were excluded from the study. We also excluded patients with end-stage renal disease requiring dialysis and patients who were already taking oral anticoagulants upon admission $(n=46)$. Finally, a total of 496 patients were included in this study (Fig. 1).

\section{MELD score and its 3 modifications}

The standard MELD [10] score and 3 modifications (MELD_XI [11], MELD_albumin [12] and MELD_ sodium [13]) were calculated according to the formulas presented in Fig. 2. If the total bilirubin or creatinine level was less than $1 \mathrm{mg} / \mathrm{dl}$, their lower limit was assumed to be $1 \mathrm{mg} / \mathrm{dl}$ to avoid negative scores.

\section{Data collection}

Data on age, sex, body mass index (BMI), history of diabetes mellitus, hypertension, heart rate, blood pressure on admission, New York Heart Association (NYHA) class, etiology of heart failure and prescribed medicine were recorded. Left ventricular ejection fraction (LVEF) was measured according to the modified Simpson's method. Pulmonary hypertension (pulmonary artery systolic pressure $\geq 50 \mathrm{mmHg}$ ), tricuspid regurgitation severity and right ventricular diastolic diameter were estimated by transthoracic echocardiography. Blood samples to test for serum sodium, potassium, albumin, creatinine, uric acid, alanine aminotransferase (ALT), aspartate aminotransaminase (AST), total bilirubin, the international normalized ratio (INR) and pro-brain natriuretic peptide (proBNP) concentrations were obtained on admission.

\section{Endpoints and follow up}

The primary endpoint was all-cause mortality. The secondary endpoint was cardiovascular mortality due to AHF. Cardiovascular mortality was coded according to the International Classification of Diseases, 10th Revision (ICD-10). All patients were followed-up in our cardiology department at 3, 6, and 12 months after discharge and then every 6 months thereafter.

\section{Statistical analysis}

Categorical variables are presented as percentages. Continuous variables are presented as the mean (standard deviation, SD) or median (interquartile) according to the distribution. Characteristics were compared according to survival status using the independent Student's t-test, the Wilcoxon test and the chi-square test, as appropriate. Comparisons between MELD and its 3 modifications were performed by the Wilcoxon test. Pairwise Spearman correlation analysis was used to investigate the relationships among MELD score and its 3 modifications.

We evaluated the association between the MELD scores and each of our outcomes using Cox proportional hazards regression with 1-SD increments in the continuously distributed MELD score and its 3 modifications. Univariate Cox regression analysis for all-cause mortality indicated that variables with statistically significant differences $(P<0.05)$ were used to derive the final model. The statistically significant variables were assessed in terms of the outcomes of all-cause mortality and cardiovascular mortality. For each outcome, we calculated unadjusted hazard ratios (HRs); HRs adjusted for age and sex (Model 1); and HRs adjusted for age, sex, NYHA class, LVEF, proBNP, systolic blood pressure and diastolic 
Assessed for eligibility $(\mathrm{n}=590)$

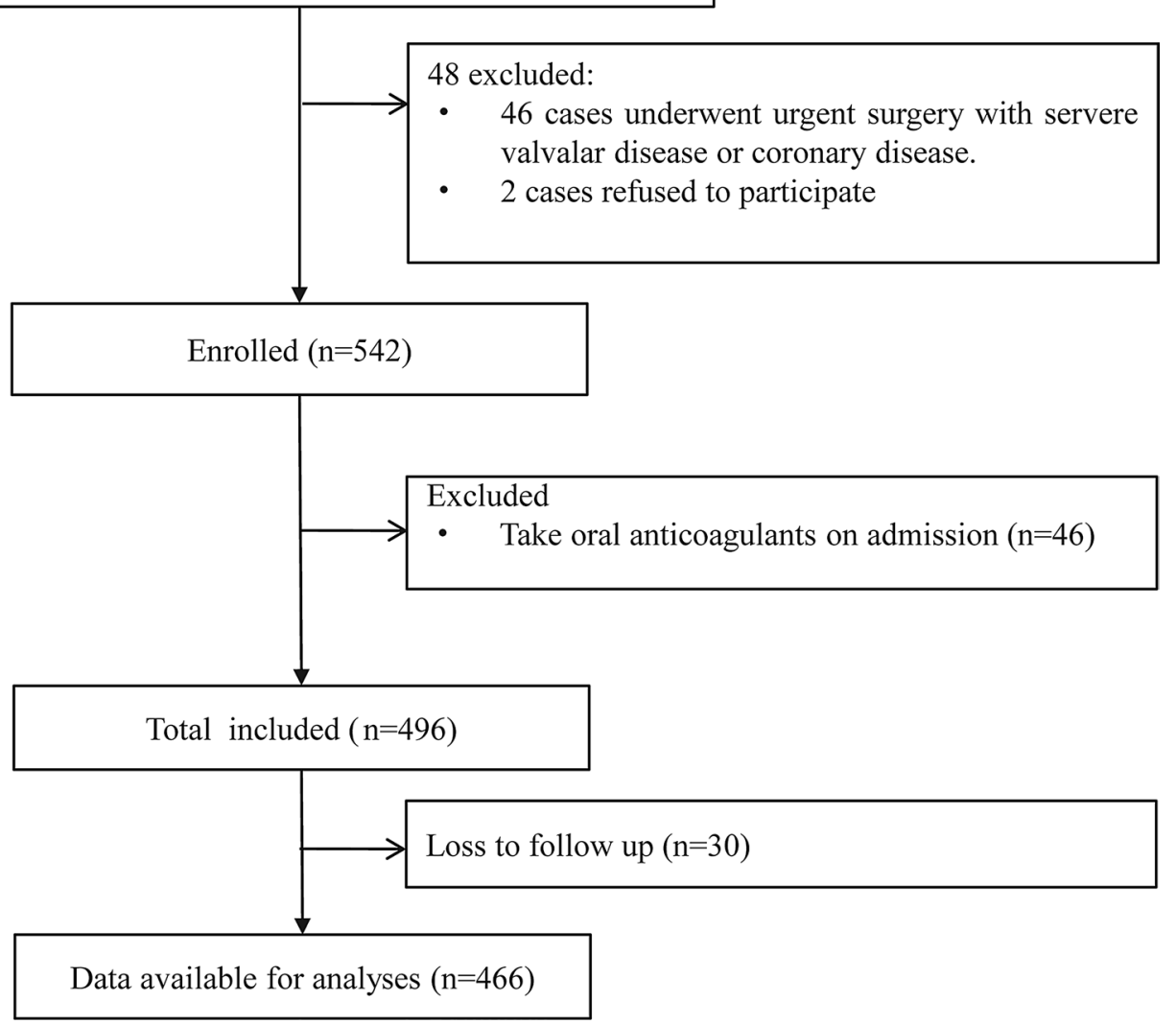

Fig. 1 Study flow chart

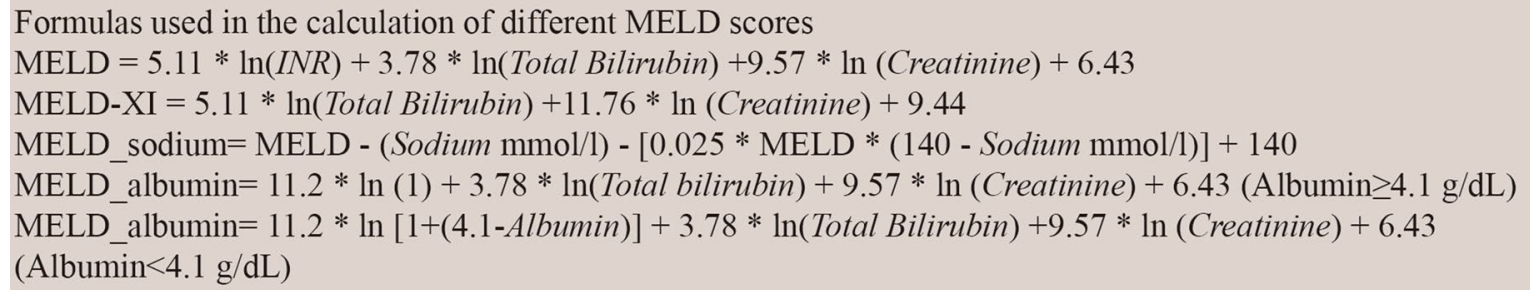

Fig. 2 The formulas for calculating the model for end-stage liver disease (MELD) score and modified MELD scores

blood pressure (Model 2) along with corresponding 95\% confidence intervals (CIs).

The predictive performance of the MELD scores was calculated according to the time-dependent receiveroperator characteristic (ROC) curves [14] and the corresponding areas under the curve (AUCs). Comparisons of the AUCs were performed with a DeLong test [15], and . Additionally, we analysed the reclassification ability of MELD and its 3 modifications using the continuous net reclassification improvement (NRI) analysis. All analyses were performed using R software version 3.6.0 (R Foundation for Statistical Computing, Vienna, Austria). Bonferroni's correction $(P<0.016$ was considered statistically significant) was applied to adjust for multiple comparisons among MELD score and its three modifications, and $P$ value $<0.05$ was considered statistically significant in other statistical analysis. 


\section{Results}

\section{Baseline characteristics}

Thirty patients were lost to follow-up. A total of 466 patients with AHF were included in the final analysis. After a median follow-up of 34 months, 196 (42.1\%) deaths occurred, and 158 (33.9\%) patients died of cardiovascular causes. At baseline, patients who died were older than survivors and were more often female. Notably, patients who died had more severe clinical symptoms, lower systolic or diastolic blood pressure, lower serum albumin, lower LVEF and higher proBNP (Table 1).

\section{Agreement of measurements}

Figure 3a presents the estimates of the MELD score and its 3 modifications using the four formulas. The median (interquartile) values of the MELD, MELD_XI,

Table 1 Baseline characteristics of the enrolled patients

\begin{tabular}{|c|c|c|c|c|}
\hline Variables & All $(n=466)$ & Deaths $(n=196)$ & Survivors $(n=270)$ & $P$ value \\
\hline Age (years) & $61.8(25.0)$ & $65.0(15.5)$ & $59.5(29.9)$ & 0.019 \\
\hline Male, \% & $314(67.4 \%)$ & $111(56.6 \%)$ & $203(75.2 \%)$ & $<0.001$ \\
\hline Diabetes, $\%$ & $118(25.3 \%)$ & $51(26.0 \%)$ & $67(24.8 \%)$ & 0.768 \\
\hline Hypertension, \% & $244(52.4 \%)$ & $97(49.5 \%)$ & 147 (54.4\%) & 0.290 \\
\hline Ischemic heart failure, $\%$ & $125(26.8 \%)$ & $53(27.0 \%)$ & $72(26.7 \%)$ & 0.928 \\
\hline NYHA & & & & 0.041 \\
\hline$\|$ & $57(21.1 \%)$ & $24(12.2 \%)$ & $81(17.4 \%)$ & \\
\hline III & $138(51.1 \%)$ & 108 (55.1\%) & $246(52.8 \%)$ & \\
\hline IV & $75(27.8 \%)$ & $64(32.7 \%)$ & 139 (29.8\%) & \\
\hline Heart rate, beats/min & $85.5(20.9)$ & $85.1(21.4)$ & $85.7(20.6)$ & 0.731 \\
\hline Systolic blood pressure (mmHg) & $127(22.6)$ & $124(19.9)$ & $129(24.2)$ & 0.020 \\
\hline Diastolic blood pressure $(\mathrm{mmHg})$ & $78.5(15.2)$ & $76.0(12.2)$ & $80.4(16.8)$ & 0.002 \\
\hline Potassium (mmol/L) & $3.99(0.51)$ & $3.99(0.51)$ & $3.99(0.50)$ & 0.912 \\
\hline Sodium (mmol/L) & $140(3.9)$ & $139(4.2)$ & $140(3.7)$ & 0.086 \\
\hline Albumin (g/L) & $3.66(0.48)$ & $3.60(0.49)$ & $3.71(0.47)$ & 0.025 \\
\hline Creatinine (mg/dL) & $1.16(0.65)$ & $1.21(0.61)$ & $1.12(0.68)$ & 0.131 \\
\hline Uric acid (mg/dL) & $8.13(2.83)$ & $8.41(3.06)$ & $7.93(2.64)$ & 0.070 \\
\hline $\operatorname{ALT}(U / L)$ & $27(17-46)$ & $26(15-50)$ & $27(18-44)$ & 0.550 \\
\hline AST (U/L) & $28(22-42)$ & $28(22-48)$ & $28(22-39)$ & 0.162 \\
\hline INR & $1.20(0.28)$ & $1.22(0.26)$ & $1.18(0.30)$ & 0.142 \\
\hline Total bilirubin (mg/dL) & $1.23(1.03)$ & $1.28(1.00)$ & $1.19(1.04)$ & 0.348 \\
\hline $\operatorname{proBNP}(\mathrm{ng} / \mathrm{L})$ & $2254(1269-5835)$ & 2873 (1572-7373) & 1779 (1087-4601) & $<0.001$ \\
\hline $\mathrm{CRP}(\mathrm{mg} / \mathrm{L})$ & $2.9(1.2-6.7)$ & $3.1(1.2-7.5)$ & $2.7(1.0-6.5)$ & 0.125 \\
\hline Anemia, \% & $133(28.5)$ & $64(32.5 \%)$ & $69(25.6 \%)$ & 0.073 \\
\hline LVEF (\%) & $41.6(14.4)$ & 39.8 (13.8) & $44.0(14.9)$ & $<0.001$ \\
\hline Pulmonary hypertension, \% & $117(25.1 \%)$ & $65(33.2 \%)$ & $52(19.3 \%)$ & 0.001 \\
\hline Tricuspid regurgitation, $\%$ & & & & 0.004 \\
\hline No & $43(9.2 \%)$ & 17 (8.7\%) & $26(9.6 \%)$ & \\
\hline Mild & 197 (42.3\%) & 70 (35.7\%) & $127(47.0 \%)$ & \\
\hline Moderate & $139(29.8 \%)$ & $57(29.1 \%)$ & $82(30.4 \%)$ & \\
\hline Severe & $87(18.7 \%)$ & $52(26.5 \%)$ & 35 (13.0\%) & \\
\hline $\operatorname{RVDd}(\mathrm{mm})$ & $39.8(8.21)$ & $40.3(8.94)$ & $39.4(7.63)$ & \\
\hline Body mass index (kg/M2) & $24.2(5.7)$ & $23.6(5.7)$ & $24.6(5.8)$ & 0.068 \\
\hline Antisterone, \% & 415 (89.1\%) & 177 (90.3\%) & $238(88.1 \%)$ & 0.451 \\
\hline ACEI/ARB, \% & $365(78.3 \%)$ & $151(77.0 \%)$ & 214 (79.3\%) & 0.566 \\
\hline Beta-blocker, \% & $373(80.0 \%)$ & $151(77.0 \%)$ & $222(82.2 \%)$ & 0.167 \\
\hline Aspirin, \% & $198(42.5 \%)$ & $85(43.4 \%)$ & 113 (41.9\%) & 0.744 \\
\hline MELD & $8.5(7.2-10.9)$ & $8.9(7.4-11.9)$ & $8.3(7.1-10.2)$ & 0.004 \\
\hline MELD_XI & $11.3(9.4-13.8)$ & $11.8(9.4-15.2)$ & $11.0(9.4-13.2)$ & 0.008 \\
\hline MELD_sodium & $8.6(6.5-12.4)$ & $9.4(6.9-14.0)$ & $8.3(6.3-11.0)$ & 0.004 \\
\hline MELD_albumin & $12.3(9.6-15.2)$ & $13.1(10.6-16.4)$ & $11.6(9.0-14.6)$ & $<0.001$ \\
\hline
\end{tabular}

Data are presented as mean (SD) or median (interquartile range), or $\mathrm{n}(\%)$. proBNP N-terminal pro-brain natriuretic peptide, NYHA New York Heart Association functional, $A L T$ alanine aminotransferase, AST aspartate aminotransaminase, LVEF left ventricular ejection fraction, RVDd right ventricular diastolic diameter, ACEI angiotensin converting enzyme inhibitors, $A R B$ angiotensin receptor blocker 


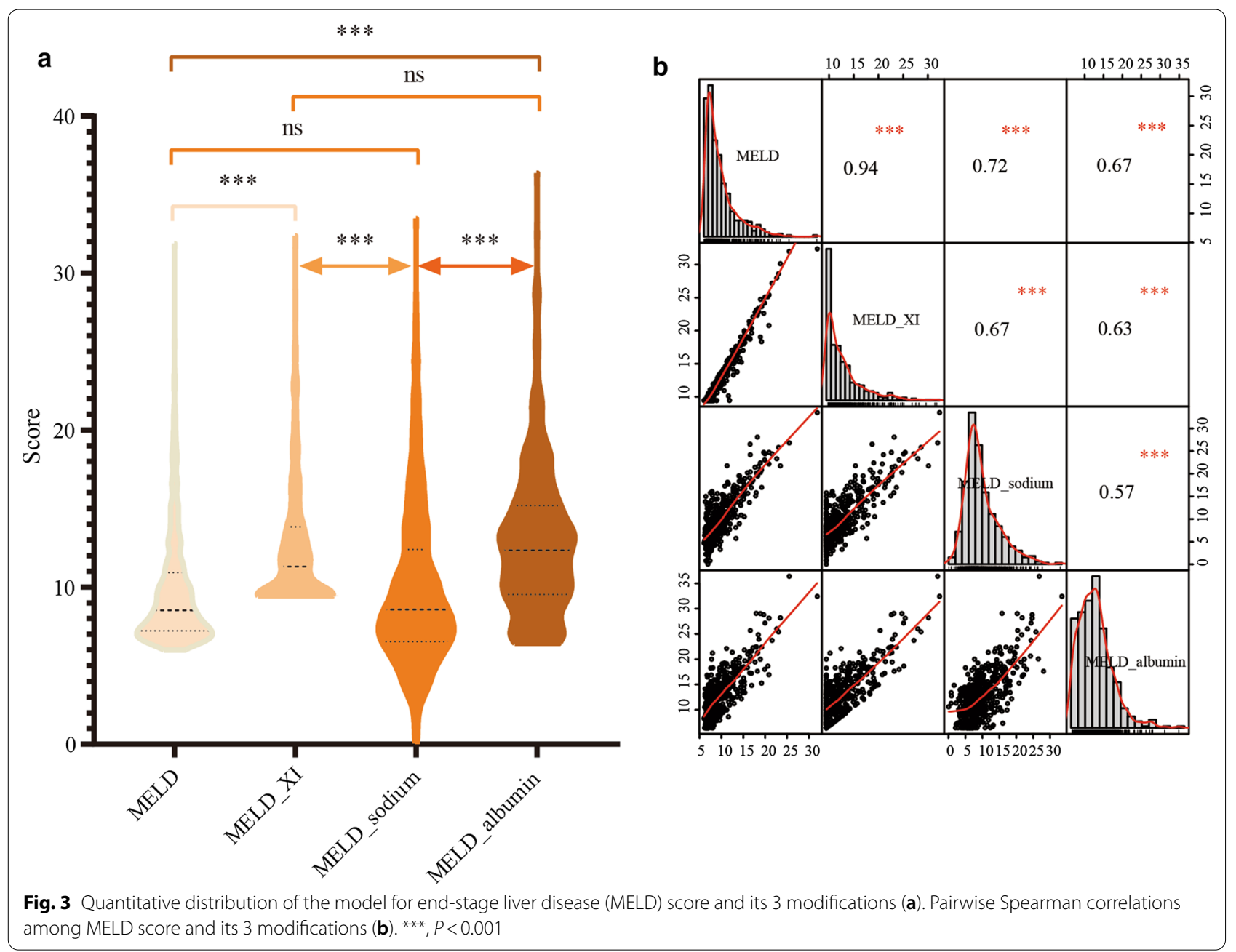

MELD_sodium and MELD_albumin scores were 7.2 (8.5-10.9), 11.3 (9.4-13.8), 8.6 (6.5-12.4) and 12.3 (9.615.2), respectively. The MELD_XI and MELD_albumin scores were significantly higher than the MELD score (all $P$ value $<0.001$ ), and the MELD_sodium score was similar to the MELD score. Pairwise Spearman correlation test was performed among the MELD score and its 3 modifications. MELD correlated better with MELD_XI ( $\mathrm{r}$ value $=0.94, P<0.001)$ than the 2 other modified MELD scores ( $\mathrm{r}$ value for MELD_sodium $=0.72, P<0.001 ; \mathrm{r}$ value for MELD_albumin $=0.67, P<0.001)$ (Fig. $3 \mathrm{~b})$.

\section{Impact of MELD score and its $\mathbf{3}$ modifications on mortality}

Table 2 shows the main predictors of all-cause mortality by univariate Cox regression analysis. Baseline age, sex, NYHA class, LVEF, proBNP, systolic blood pressure, diastolic blood pressure and pulmonary hypertension. $(P$ values $<0.05$ ) were considered potential risk factors for long-term all-cause mortality in patients with AHF. After further adjusting all risk factors at baseline, standardized hazard ratios (HRs) with 95\% confidence intervals (CIs) expressing the risk of all-cause mortality were 1.22 (1.06-1.40), 1.20 (1.04-1.39), 1.23 (1.06-1.42) and 1.21 (1.05-1.41) for the MELD, MELD_ XI, MELD_sodium and MELD_albumin scores, respectively, and the corresponding hazard ratios for cardiovascular mortality were 1.30 (1.12-1.50), 1.28 (1.10-1.49), 1.34 (1.14-1.57) and 1.27 (1.07-1.49), respectively (Table 3 ).

\section{Prognostic accuracy}

The predictive accuracy of the MELD, MELD_XI, MELD_ sodium and MELD_albumin scores was compared using time-dependent ROC curves. If we considered the entire follow-up period, the MELD_albumin score showed the best prognostic accuracy for all-cause and cardiovascular mortality in nearly the entire follow-up period (Figs. 4a, $5 \mathrm{a})$. The quantified AUCs with a four-year cut-off for allcause mortality outcome were $0.580(0.509-0.650)$ for the MELD score, $0.544(0.476-0.614)$ for the MELD_XI score, 0.590 (0.519-0.661) for the MELD_sodium score, 
Table 2 Univariate analysis of predictors for all-cause mortality

\begin{tabular}{|c|c|c|}
\hline & $\mathrm{HR}(95 \% \mathrm{Cl})$ & $P$ value \\
\hline Age (per year increase) & $1.00(1.00,1.01)$ & 0.016 \\
\hline Male & $0.55(0.41,0.73)$ & $<0.001$ \\
\hline Diabetes & $1.01(0.74,1.39)$ & 0.936 \\
\hline Hypertension & $0.87(0.66,1.15)$ & 0.327 \\
\hline Ischemic heart failure & $1.12(0.82,1.54)$ & 0.483 \\
\hline \multicolumn{3}{|l|}{ NYHA } \\
\hline$\|$ & 1.00 & \\
\hline III & $1.63(1.05,2.54)$ & 0.031 \\
\hline IV & $2.01(1.25,3.21)$ & 0.004 \\
\hline Heart rate (per beats/min increase) & $1.00(0.99,1.00)$ & 0.521 \\
\hline Systolic blood pressure (per mmHg increase) & $0.99(0.99,1.00)$ & 0.023 \\
\hline Diastolic blood pressure (per mmHg increase) & $0.98(0.97,0.99)$ & 0.001 \\
\hline Potassium (per mmol/L increase) & $1.08(0.81,1.42)$ & 0.612 \\
\hline Uric acid (per mg/dL increase) & $1.05(0.99,1.10)$ & 0.057 \\
\hline ALT (per U/L increase) & $1.00(1.00,1.00)$ & 0.598 \\
\hline AST (per U/L increase) & $1.00(1.00,1.00)$ & 0.659 \\
\hline proBNP (per ng/L increase) & $1.00(1.00,1.00)$ & $<0.001$ \\
\hline CRP (per mg/L increase) & $1.01(0.99,1.03)$ & 0.082 \\
\hline Anemia & $1.22(0.82,1.80)$ & 0.325 \\
\hline LVEF (per 1 unit increase) & $0.98(0.97,0.99)$ & 0.001 \\
\hline Pulmonary hypertension & $1.69(1.26,2.28)$ & 0.001 \\
\hline \multicolumn{3}{|l|}{ Tricuspid regurgitation } \\
\hline No & 1.00 & \\
\hline Mild & $0.96(0.54,1.71)$ & 0.890 \\
\hline Moderate & $1.16(0.65,2.09)$ & 0.619 \\
\hline Severe & $1.74(0.96,3.14)$ & 0.068 \\
\hline RVDd (per mm increase) & $1.02(1.00,1.04)$ & 0.056 \\
\hline Body mass index (per kg/M2 increase) & $0.98(0.95,1.00)$ & 0.082 \\
\hline Antisterone & $1.13(0.70,1.81)$ & 0.622 \\
\hline ACEI/ARB & $0.94(0.67,1.31)$ & 0.699 \\
\hline Beta-blocker & $0.79(0.57,1.10)$ & 0.163 \\
\hline Aspirin & $1.08(0.82,1.44)$ & 0.583 \\
\hline
\end{tabular}

NYHA New York Heart Association functional class, ALT alanine aminotransferase, AST aspartate aminotransaminase, LVEF left ventricular ejection fraction, $A C E I$ angiotensin converting enzyme inhibitors, $A R B$ angiotensin receptor blocker

and $0.658(0.591-0.728)$ for the MELD_albumin score. The MELD_albumin score was significantly better than the MELD and MELD_XI for predicting 4-year all-cause mortality risk (all $P$ value $<0.016$, Fig. 4 b).

The quantified AUCs with a four-year cut-off for cardiovascular mortality outcome were 0.617 (0.545-0.689) for the MELD score, $0.580(0.508-0.652)$ for the MELD_ XI score, $0.625(0.553-0.697)$ for the MELD_sodium score, and $0.663(0.593-0.734)$ for the MELD_albumin score. The MELD_albumin score was significantly better than the MELD_XI score for predicting the 4-year risk of cardiovascular mortality $(P$ value $=0.009$, Fig. $5 b)$.
However, MELD_albumin showed no significant better than the MELD and MELD_sodium score in predicting 4-year cardiovascular mortality risk.

These findings were evaluated by the NRI analysis (Table 4). MELD_albumin score increased the NRI over and beyond MELD (13.1\%, $P=0.003)$, MELD_XI (14.8\%, $P=0.006)$, and MELD_sodium $(11.9 \%, P=0.006)$ scores for all-cause mortality. However, MELD_albumin did not increase NRI in the reclassification of cardiovascular mortality.

\section{Discussion}

Our study demonstrated that liver and renal function evaluated by the MELD_albumin score could serve as an important predictor of the prognosis of AHF. Our study also indicates for the first time that evaluating liver and renal function using the MELD_albumin score outperforms evaluation using MELD and other modified MELD scores to discriminate the risk of all-cause mortality in AHF patients. The present data extend the usefulness of modified MELD scores to predict clinical events in patients with AHF.

Our findings are consistent with previous studies reporting the prognostic role of liver and renal function on mortality in patients with AHF $[3,6,7]$ and add interesting information to the available literature on this topic. The MELD score and its modifications, which have been widely used as prognostic predictors in patients with liver diseases $[10,12]$, have recently been shown to be associated with worse clinical outcomes in patients with AHF $[3,6,7]$. In the study conducted by Kim et al. [6], elevated MELD, MELD_XI, and MELD_sodium scores were associated with an increased risk for composite endpoint of death, heart transplantation and ventricular-assist device requirement in ambulatory patients with heart failure (HR was 1.10 (1.06-1.14) for MELD; HR was 1.13 (1.071.19) for MELD_XI; HR was 1.10 (1.06-1.14) for MELD_ sodium). Another study by Biegus et al. [3] demonstrated that the MELD or MELD_XI score at baseline was predictive of 1-year all-cause mortality in patients with AHF (HR was 1.08 (1.02-1.35) for MELD, HR 1.11 (1.05-1.2) for MELD_XI). Similar results were also found in another study by Biegus et al. [7], in which the MELD-XI score was significantly associated with all-cause death (HR 1.11 (1.04-1.17)). Notably, the MELD score has been shown to identify risk in patients supported with a left ventricular assist device [16] and patients who underwent cardiac surgery [17].

Compared to the previous studies, the difference in hazard ratios of our study may arise from the relatively larger sample size, the longer follow-up duration, the value of the MELD score or its modifications, the definition of the outcome and the cofounding factors we 
Table 3 Standardized hazard ratios (95\% confidence intervals) of mortality

\begin{tabular}{|c|c|c|c|c|c|c|}
\hline & \multicolumn{2}{|l|}{ Unadjusted } & \multicolumn{2}{|l|}{ Model 1} & \multicolumn{2}{|l|}{ Model 2} \\
\hline & $\mathrm{HR}(95 \% \mathrm{Cl})$ & $P$ value & $\mathrm{HR}(95 \% \mathrm{Cl})$ & $P$ value & $\mathrm{HR}(95 \% \mathrm{Cl})$ & $P$ value \\
\hline \multicolumn{7}{|c|}{ All cause mortality $(196 / 466,42.1 \%)$} \\
\hline MELD & $1.23(1.10-1.38)$ & $<0.001$ & $1.31(1.16-1.47)$ & $<0.001$ & $1.21(1.05-1.40)$ & 0.008 \\
\hline MELD_XI & $1.21(1.08-1.36)$ & 0.002 & $1.28(1.14-1.45)$ & $<0.001$ & $1.20(1.04-1.39)$ & 0.014 \\
\hline MELD_sodium & $1.29(1.14-1.46)$ & $<0.001$ & $1.36(1.19-1.54)$ & $<0.001$ & $1.23(1.06-1.43)$ & 0.005 \\
\hline MELD_albumin & $1.27(1.13-1.42)$ & $<0.001$ & $1.31(1.16-1.47)$ & $<0.001$ & $1.22(1.05-1.41)$ & 0.012 \\
\hline \multicolumn{7}{|c|}{ Cardiovascular mortality (158/466, 33.9\%) } \\
\hline MELD & $1.31(1.17-1.48)$ & $<0.001$ & $1.39(1.23-1.56)$ & $<0.001$ & $1.29(1.11-1.50)$ & 0.001 \\
\hline MELD_XI & $1.29(1.14-1.46)$ & $<0.001$ & $1.36(1.20-1.53)$ & $<0.001$ & $1.27(1.09-1.48)$ & 0.002 \\
\hline MELD_sodium & $1.41(1.23-1.60)$ & $<0.001$ & $1.48(1.29-1.69)$ & $<0.001$ & $1.34(1.14-1.56)$ & $<0.001$ \\
\hline MELD_albumin & $1.31(1.15-1.48)$ & $<0.001$ & $1.35(1.18-1.53)$ & $<0.001$ & $1.27(1.07-1.50)$ & 0.006 \\
\hline
\end{tabular}

Model 1 was adjusted for age and sex

Model 2 was adjusted age, sex, NYHA class, LVEF, proBNP, systolic blood pressure, diastolic blood pressure and pulmonary hypertension

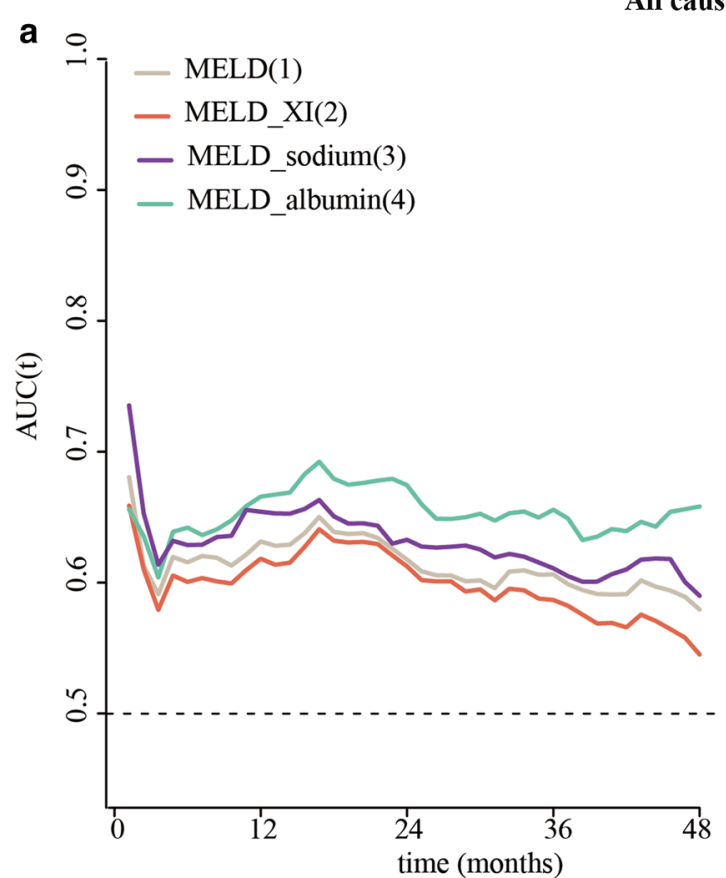

Fig. 4 Time-dependent ROC analysis (a) and areas under the curve (b) of the model for end-stage liver disease (MELD) score and its 3 modifications for all-cause mortality

adjusted. Notably, our study not only highlighted the prognostic role of the modified MELD score but also indicated that the MELD_albumin score was superior to the MELD and other modified MELD scores in predicting the risk of mortality. Our study supports the incorporation of serum albumin into the modified MELD score to provide additional risk information in patients with AHF.

The underlying mechanism is that venous congestion and hypoperfusion can contribute to elevated liver enzymes and creatinine, resulting in cardio-renalhepatic syndrome [18]. The progression of liver dysfunction in patients with AHF is complex and may follow any of several pathways. Biochemical markers of cholestasis, such as $\gamma$-glutamyl transpeptidase, bilirubin, and alkaline phosphatase, are increased in the plasma of AHF patients with elevated central venous pressure [19]. Hypoperfusion-induced hypoxic liver injury is also the major cause of massively elevated aminotransferase levels in patients with AHF, and the elevation of these levels is a risk factor 
a

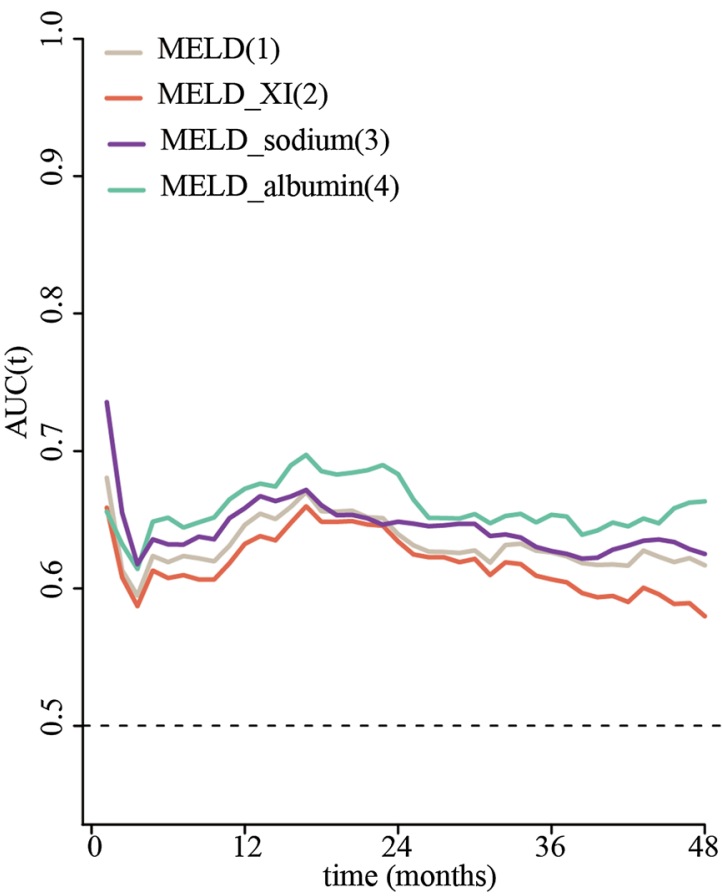

Cardiovascular mortality

b

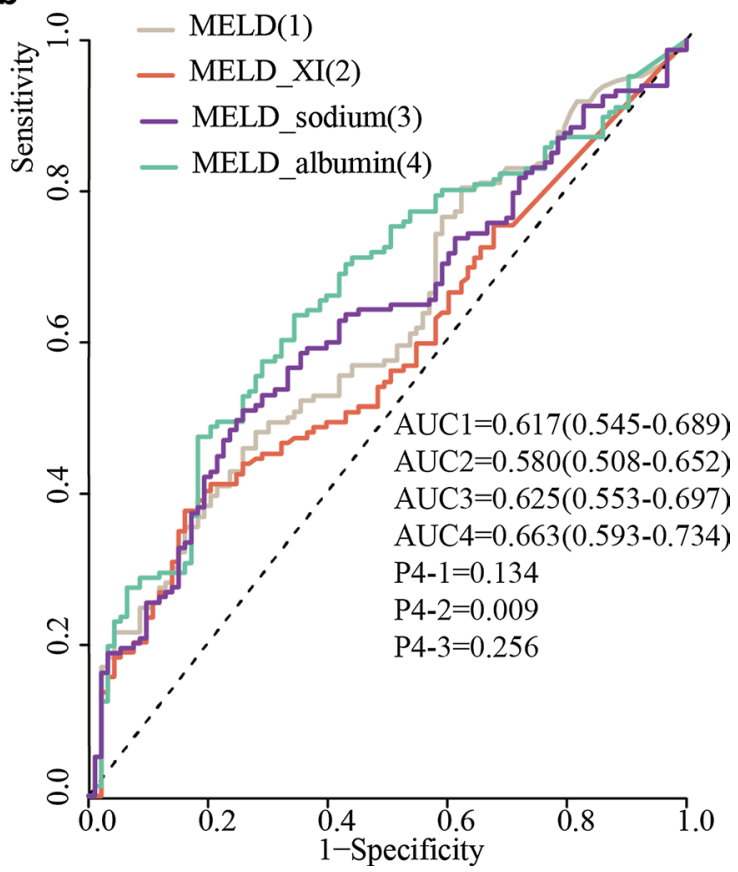

Fig. 5 Time-dependent ROC analysis (a) and areas under the curve (b) of the model for end-stage liver disease (MELD) score and its 3 modifications for cardiovascular mortality

Table 4 Net reclassification improvement by comparing different MELD equation

\begin{tabular}{|c|c|c|c|c|c|c|}
\hline & \multicolumn{3}{|c|}{ All-cause mortality } & \multicolumn{3}{|c|}{ Cardiovascular mortality } \\
\hline & NRI (\%) & $\mathrm{Cl}(95 \%)$ & $P$ & NRI (\%) & $\mathrm{Cl}(95 \%)$ & $P$ \\
\hline MELD_albumin vs MELD & 13.1 & 5.2 to 22.8 & 0.003 & 2.5 & -8.9 to 9.9 & 0.595 \\
\hline MELD_albumin vs MELD_XI & 14.8 & 5.5 to 21.6 & 0.002 & 3.1 & -8.3 to 11.9 & 0.539 \\
\hline MELD_albumin vs MELD_sodium & 11.9 & 4.6 to 21.9 & 0.006 & 3.6 & -9.0 to 14.9 & 0.547 \\
\hline
\end{tabular}

NRI net reclassification improvement, $C /$ confidence interval

for mortality [20]. In the setting of renal dysfunction, congestion was a stronger predictor of worsening renal function than cardiac output or mean arterial pressure in patients with AHF [21, 22]. Congestion is the main profile of diastolic dysfunction. Interestingly, a study also indicated that renal insufficiency is more important in determining the risk of mortality in patients with diastolic cardiac dysfunction than in those with systolic dysfunction [23]. The increase in central venous pressure is associated with worsen renal function through several mechanisms including the decrease in renal blood flow and interstitial fibrosis [24].
There are several limitations in our study. First, the empirically chosen Chinese population was a major limitation. Validation in other population should be performed to verify our results. Second, although we included a set of confounding factors and indicated the strong prognostic power of the MELD score and its modifications in patients with AHF, unmeasured cofounding factors may also play a role. Finally, the MELD score or its modifications are subject to laboratory variations, and previous oral medications such as diuretics can impact sodium and creatinine excretion, which could confound the results. 


\section{Conclusions}

Quantifying liver and renal function using the MELD_ albumin score in patients with acute heart failure assesses the risk of mortality more accurately than the commonly used MELD score or its other modifications.

\begin{abstract}
Abbreviations
AHF: Acute heart failure; ALT: Alanine aminotransferase; AST: Aspartate aminotransaminase; AUC: Areas under the curve; BMI: Body mass index; Cl: Confidence interval; HF: Heart failure; HR: Hazard ratio; INR: International normalized ratio; LVEF: Left ventricular ejection fraction; MELD: Model for end-stage liver disease; MELD_XI: MELD excluding the international normalized ratio; MELD_ sodium: MELD including sodium; NRI: Net reclassification improvement: NYHA: New York Heart Association; proBNP: Pro-brain natriuretic peptide; ROC: Receiver operating characteristic.
\end{abstract}

\section{Acknowledgements}

Not applicable.

\section{Authors' contributions}

SGL and XLL conceived and supervised the study; XYL, IC, XZ and TY were responsible for data collection. WMY and HFZ were responsible for analysis of data; SGL drafted the manuscript; HFZ and XLL made manuscript revisions. All authors read and approved the final manuscript.

\section{Funding}

The study was supported by the Twelve-Fifth National Key Technology R\&D Program (2011BAl11B08). The funding bodies played no role in the design of the study and collection, analysis, and interpretation of data and in writing the manuscript.

\section{Availability of data and materials}

The datasets used and/or analyzed during the current study are available from the corresponding author on reasonable request.

\section{Declarations}

\section{Ethics approval and consent to participate}

The study was approved by the ethics committee of the First Affiliated Hospital of Nanjing Medical University, and all patients provided written informed consent. The First Affiliated Hospital of Nanjing Medical University granted access to the personal data in the study. All procedures conformed to the standards of the Declaration of Helsinki.

\section{Consent for publication}

Not applicable.

\section{Competing interests}

The authors have no conflict of interest to disclose.

Received: 27 October 2020 Accepted: 28 February 2021

Published online: 09 March 2021

\section{References}

1. Benjamin EJ, Blaha MJ, Chiuve SE, Cushman M, Das SR, Deo R, et al. Heart disease and stroke statistics-2017 update: a report from the American Heart Association. Circulation. 2017;135:e146-603.

2. Dunlay SM, Redfield MM, Weston SA, Therneau TM, Hall Long K, Shah ND, et al. Hospitalizations after heart failure diagnosis a community perspective. J Am Coll Cardiol. 2009;54:1695-702.

3. Biegus J, Zymlinski R, Sokolski M, Siwolowski P, Gajewski P, NawrockaMillward S, et al. Impaired hepato-renal function defined by the MELD XI score as prognosticator in acute heart failure. Eur J Heart Fail. 2016;18:1518-21.
4. Weidmann ZM, Breidthardt T, Twerenbold R, Zusli C, Nowak A, von Eckardstein $A$, et al. Prediction of mortality using quantification of renal function in acute heart failure. Int J Cardiol. 2015;201:650-7.

5. Shinagawa H, Inomata T, Koitabashi T, Nakano H, Takeuchi I, Naruke T, et al. Prognostic significance of increased serum bilirubin levels coincident with cardiac decompensation in chronic heart failure. Circ J. 2008;72:364-9.

6. Kim MS, Kato TS, Farr M, Wu C, Givens RC, Collado E, et al. Hepatic dysfunction in ambulatory patients with heart failure: application of the MELD scoring system for outcome prediction. J Am Coll Cardiol. 2013;61:2253-61.

7. Biegus J, Demissei B, Postmus D, Cotter G, Davison BA, Felker GM, et al. Hepatorenal dysfunction identifies high-risk patients with acute heart failure: insights from the RELAX-AHF trial. ESC Heart Fail. 2019;6:1188-98.

8. Grodin JL, Gallup D, Anstrom KJ, Felker GM, Chen HH. Tang WHW Implications of Alternative Hepatorenal Prognostic Scoring Systems in Acute Heart Failure (from DOSE-AHF and ROSE-AHF). Am J Cardiol. 2017:119:2003-9.

9. Ponikowski P, Voors AA, Anker SD, Bueno H, Cleland JGF, Coats AJS, et al. 2016 ESC Guidelines for the diagnosis and treatment of acute and chronic heart failure: The Task Force for the diagnosis and treatment of acute and chronic heart failure of the European Society of Cardiology (ESC)Developed with the special contribution of the Heart Failure Association (HFA) of the ESC. Eur Heart J. 2016;37:2129-200.

10. Kamath PS, Wiesner RH, Malinchoc M, Kremers W, Therneau TM, Kosberg $C L$, et al. A model to predict survival in patients with end-stage liver disease. Hepatology. 2001;33:464-70.

11. Heuman DM, Mihas AA, Habib A, Gilles HS, Stravitz RT, Sanyal AJ, et al. MELD-XI: a rational approach to "sickest first" liver transplantation in cirrhotic patients requiring anticoagulant therapy. Liver Transpl. 2007:13:30-7.

12. Chokshi A, Cheema FH, Schaefle KJ, Jiang J, Collado E, Shahzad K, et al. Hepatic dysfunction and survival after orthotopic heart transplantation: application of the MELD scoring system for outcome prediction. J Heart Lung Transplant. 2012;31:591-600.

13. Kim WR, Biggins SW, Kremers WK, Wiesner RH, Kamath PS, Benson JT, et al. Hyponatremia and mortality among patients on the liver-transplant waiting list. N Engl J Med. 2008;359:1018-26.

14. Heagerty PJ, Lumley T. Pepe MS Time-dependent ROC curves for censored survival data and a diagnostic marker. Biometrics. 2000;56:337-44.

15. DeLong ER, DeLong DM. Clarke-Pearson DL Comparing the areas under two or more correlated receiver operating characteristic curves: a nonparametric approach. Biometrics. 1988;44:837-45.

16. Yalcin YC, Muslem R, Veen KM, Soliman OI, Manintveld OC, Darwish Murad S, et al. Impact of preoperative liver dysfunction on outcomes in patients with left ventricular assist devices. Eur J Cardiothorac Surg. 2019:57:920-8.

17. Hawkins RB, Young BAC, Mehaffey JH, Speir AM, Quader MA, Rich JB, et al. Model for end-stage liver disease score independently predicts mortality in cardiac surgery. Ann Thorac Surg. 2019;107:1713-9.

18. Harjola VP, Mullens W, Banaszewski M, Bauersachs J, Brunner-La Rocca $\mathrm{HP}$, Chioncel $\mathrm{O}$, et al. Organ dysfunction, injury and failure in acute heart failure: from pathophysiology to diagnosis and management. A review on behalf of the Acute Heart Failure Committee of the Heart Failure Association (HFA) of the European Society of Cardiology (ESC). Eur J Heart Fail. 2017;19:821-36.

19. van Deursen VM, Damman K, Hillege HL, van Beek AP, van Veldhuisen DJ. Voors AA Abnormal liver function in relation to hemodynamic profile in heart failure patients. J Card Fail. 2010;16:84-90.

20. Whitehead MW, Hawkes ND, Hainsworth I. Kingham JG A prospective study of the causes of notably raised aspartate aminotransferase of liver origin. Gut. 1999;45:129-33.

21. Damman K, van Deursen VM, Navis G, Voors AA, van Veldhuisen DJ, Hillege HL. Increased central venous pressure is associated with impaired renal function and mortality in a broad spectrum of patients with cardiovascular disease. J Am Coll Cardiol. 2009;53:582-8.

22. Mullens W, Abrahams Z, Francis GS, Sokos G, Taylor DO, Starling RC, et al. Importance of venous congestion for worsening of renal function in advanced decompensated heart failure. J Am Coll Cardiol. 2009:53:589-96. 
23. Ahmed A, Rich MW, Sanders PW, Perry GJ, Bakris GL, Zile MR, et al. Chronic kidney disease associated mortality in diastolic versus systolic heart failure: a propensity matched study. Am J Cardiol. 2007;99:393-8.

24. Legrand M, Mebazaa A, Ronco C, Januzzi JL Jr. When cardiac failure, kidney dysfunction, and kidney injury intersect in acute conditions: the case of cardiorenal syndrome. Crit Care Med. 2014;42:2109-17.

\section{Publisher's Note}

Springer Nature remains neutral with regard to jurisdictional claims in published maps and institutional affiliations.
Ready to submit your research? Choose BMC and benefit from:

- fast, convenient online submission

- thorough peer review by experienced researchers in your field

- rapid publication on acceptance

- support for research data, including large and complex data types

- gold Open Access which fosters wider collaboration and increased citations

- maximum visibility for your research: over 100M website views per year

At BMC, research is always in progress.

Learn more biomedcentral.com/submissions 